\title{
Development of mathematical model for multi-user coded-cooperation based cognitive radio system and its outage probability analysis
}

Garima Singh ( $\sim$ garimasinghmavi@gmail.com )

Delhi Technological University https://orcid.org/0000-0001-6954-2324

\section{Gurjit Kaur}

Delhi Technological University

\section{Research Article}

Keywords: Coded-cooperation, Cognitive radio, Single relay, Multi-user, Interference temperature constraint, Outage probability.

Posted Date: June 1st, 2021

DOI: https://doi.org/10.21203/rs.3.rs-380183/v1

License: (c) (1) This work is licensed under a Creative Commons Attribution 4.0 International License.

Read Full License

Version of Record: A version of this preprint was published at Wireless Personal Communications on November 29th, 2021. See the published version at https://doi.org/10.1007/s11277-021-09247-9. 


\title{
Development of mathematical model for multi-user coded-cooperation based cognitive radio system and its outage probability analysis
}

\author{
Garima Singh*, Dr.Gurjit Kaur \\ Department of Electronics and Communication \\ Delhi Technological University, Delhi, India. \\ Email:garimasinghmavi@gmail.com*, gurjeet_kaur@rediffmail.com
}

\begin{abstract}
In this paper, single and multi-user codedcooperation based cognitive radio system is developed by designing its mathematical model where both source and relay will communicate to a single destination with the help of each other. Then all possible multi-user scenarios are developed and their end-to-end outage probability (Pout) is calculated for underlay mode of cognitive radio. The performance of the system is analyzed in the form of channel gain and interference temperature constraint for Rayleigh fading channel. The proposed system concludes that the coded cooperation with cognitive radio outperform the available techniques in the form of bandwidth, diversity, spectrum utilization efficiency and also improves the quality of communication. Furthermore, the theoretical analysis of the outage probability for both system models is validated by asymptotic analysis. The proposed system can set as a standard for all those cognitive radio applications which requires better spectrum efficiency even if there is a scarcity of multiple physical antennas.
\end{abstract}

Keywords: Coded-cooperation, Cognitive radio, Single relay, Multi-user, Interference temperature constraint, Outage probability.

\section{I.Introduction}

Today's biggest challenge is to fulfill the spectrum requirements of the next generation bandwidthhungry wireless applications. The only possible approach is to utilize the existing frequency spectrum more smartly. For such type of applications cognitive radio (CR) is the best solution which offers an optimized way of spectrum allocation [1]-[4]. This is because, it can be configured and programmed to use the best possible vacant frequency spectrum in its vicinity which leads to utilization of the same spectrum in a much efficient way [5], [6]. Next-generation wireless applications are not limited to point-to-point or point-to-multipoint applications but require wide coverage through wireless communication. Also, these applications require transmission diversity which can be achieved by using more than one antenna [7]. But, the next-generation wireless system can support only one antenna due to their small size and less complex architecture.

In recent times, a new technique i.e. cooperative communication has been proposed in which a single antenna can work as a relay as well as virtual antenna and create a useful multi-antenna environment. This relay will generate multiple replicas of the transmitted signal which ultimately provides transmission diversity. So, cooperative communication can provide high spectral efficiency, wide network coverage even with minimum outage probability. It has many types of cooperative protocols like amplify-and-forward (A\&F), decodeand-forward (D\&F), and coded cooperation (CC), etc. [17]. Among all these protocols, the coded cooperation is the only bandwidth-efficient technique because it sends some additional redundancy bits in its user's data for partner or relay and avoids repetition of received bits.

In this research paper, we are going to propose a hybrid system where the benefits of coded cooperation have been exploited and merged with cognitive radio which ultimately developed a spectrum as well as bandwidth-efficient system.

Many researchers are working on cooperative communication as well as on cognitive radio 
systems. In [8], an A\&F relay selection technique was discussed for underlay based cognitive radio network. In [9] and [10], the working of A\&F based cooperative relaying system in spectrum sharing mode has been evaluated. While in [11], A\&F based multi-hop system in spectrum-sharing mode was analyzed. Exact outage probability has been calculated for partial $A \& F$ based relay selection scheme by Chen et al. in [12] for cognitive relay networks with imperfect CSI. In [13], the Zhong et al. had analyzed the outage capacity of D\&F based dual-hop cognitive systems under Nakagami-m fading. But, the above mentioned cooperative schemes are not much bandwidth-efficient and also have transmission diversity constraint. In [14], Moualeu et al. have calculated the BER for underlay based cognitive coded cooperation system under interference power constraint using turbo codes. The above literature has shown that all the researchers have mainly focused on the system where the only secondary user can send its data over the channel through relay. In [15], we already proposed the cognitive radio-based multi-relay coded cooperation concept in which data of only secondary source is transmitted with the help of relay. The issue of the system was that the relay could not transmit its own data when the source was utilizing it as a relay. So, this work is an extension of our previous work [15] where a multi-user communication system is proposed in which both the source and relay can transmit their data simultaneously to the single destination by acting as a relay to each other without any interference.

The proposed system efficiently utilize the channel resources in a practical manner where the coded cooperation is used as cooperative relaying protocol. A single relay-based cognitive radio system is developed which is further extended to an advanced multi-user system. For the rigorous analysis of an advanced multi-user system, we have developed four different scenarios with their mathematical model explaining the secondary user and relay transmission process. In the proposed model, the code-word of each user is partitioned using coded cooperation and the portions of these code-words are transmitted independently over the fading channels by utilizing minimum bandwidth. Here, an end-toend outage probability for all possible scenarios is calculated to conclude about the system model performance which outperforms the existing techniques in the form of channel gain and outage probability. Along with this, we have also worked on a high signal-to-noise ratio (SNR) regime to validate the research asymptotically.

This research paper is outlined in a manner that, section II contains the introduction of the system model. In section III, the outage probability of the primary model for cognitive radio based coded cooperation is analytically derived and extended by an enhanced model in section IV with high SNR discussion. Moreover, the casewise outage probability of multi-user transmission scenario is explained in section V. Numerical results with discussion for the proposed system are provided in section VI followed by the conclusion.

\section{II.System Model}

In this research work, a hierarchical mathematical model for coded cooperation based cognitive radio system is proposed where source and relay cooperate with each other by transmitting their bits over the two consecutive bit periods. The bits can be transmitted by a user during the first interval and the partner can detect and retransmit it again during the second interval. The system model consists of three nodes i.e.(i) licensed/primary node $p$, (ii) unlicensed/secondary source node $s$ and (iii) secondary destination node $d$. Secondary source and secondary destination are communicating with each other directly and also through coded cooperation based relay node symbolize by $r$ as shown in Fig.1. One way of limiting the use of the primary user's spectrum by the secondary user is by incorporating an interference threshold i.e. interference temperature constraint, $Q$ at the primary user. The interference temperature constraint corresponds to the maximum interference power level allowed by the primary node for transmitting the data of secondary node and this method of using spectrum of primary user is called underlay mode of cognitive radio. The transmitter $i \in\{s, r, 1,2\}$ and the receiver 
$j \in\{d, p, r, 1,2\}$ are using Rayleigh flat faded independent and identically distributed (i.i.d.) channel of gain $g_{i, j}$, for their transmission having zero-mean and unit variance. This channel gain is inversely proportional to average channel gain, $\left|h_{i, j}\right|^{2}$ and distributed exponentially with parameter $\lambda_{i, j}$. The transmitted power $P_{t}$ between any two nodes is adjusted $P_{t}\left|g_{i, j}\right| \leq Q$ to remain up to the level of interference constraint, $Q$ [15]. Therefore, $Q / g_{i, j}$ is the maximum allowable instantaneous power of secondary source and relay node.

Every secondary source message is encoded into a rate $R_{r}$ of code-word of length $L$, represented as $y=\{y[0], \ldots \ldots, y[L-1]\}^{T}$. Two phases of cooperative communication are used to transmit this code-word. In Phase I, first $L_{1}$ symbols of code-word i.e. $y^{(1)}=\{y[0], \ldots . . ., y[L-1]\}^{T}$ are transmitted and are encoded by the rate $R_{1}>R_{r}$ while the remaining portion of the code-word i.e. $y^{(2)}=\left\{y\left[L_{1}\right], \ldots \ldots, y\left[L_{1}+L_{2}-1\right]\right\}^{T}$ with $\quad L_{2}=L-L_{1}$ symbols will be decoded automatically by using the redundancy bits sent along with the $y^{(1)}$. The integration of $y^{(1)}$ with $y^{(2)}$ as one, will give a dominant code-word of rate $R_{r}$.

In the first phase, source will transmit the whole encoded code-word to the destination but the relay will just listen to the first portion of code-word i.e. $y^{(1)}$ with a code rate of $R_{1}$ and this signal will be received at relay node given by equation (1) and (2) respectively,

$x_{r}[n]=h_{s, r} \sqrt{P_{t}} y[n]+w_{r}[n]$,

$x_{d}^{(1)}[n]=h_{s, d} \sqrt{P_{t}} y[n]+w_{d}[n]$.

Where, $\quad n=0, \ldots \ldots . . ., L_{1}-1 \quad$ and $\quad w_{r}$ and $w_{d}$ are Additive White Gaussian Noise (AWGN) at the relay node and secondary destination respectively. At this point, we define $\beta=L_{1} / L$ such that Phase I code rate will be $R_{1}=R_{r} / \beta$. The relay node is competent enough to effectively decode the remaining $L_{2}$ symbols i.e. $y^{(2)}$ of the code-word and transmit that to the secondary destination successfully in Phase II, which is stated as

$$
x_{d}^{(2)}[n]=\left\{\begin{array}{l}
h_{r, d} \sqrt{P_{t}} y\left[L_{1}+n\right]+w^{(2)} d[n], i f \delta \geq 2 \\
h_{s, d} \sqrt{P_{t}} y\left[L_{1}+n\right]+w^{(2)} d[n] \text { otherwise }
\end{array}\right\}
$$

where, $n=0, \ldots \ldots \ldots, L_{2}-1$. The message at the secondary destination will be decoded on the basis of integration of symbols received in phase I and II, i.e., $x_{d}=\left[\left(x_{d}^{(1)}\right)^{T}\left(x_{d}^{(2)}\right)^{T}\right]^{T}$,

where,

$$
\begin{gathered}
x_{d}^{(1)}=\left[x_{d}^{(1)}(0) \ldots \ldots \ldots \ldots \ldots \ldots \ldots x_{d}^{(1)}\left(L_{1}-1\right)\right]^{T}, \\
x_{d}^{(2)}=\left[x_{d}^{(2)}(0) \ldots \ldots \ldots \ldots \ldots \ldots x_{d}^{(2)}\left(L_{2}-1\right)\right]^{T} .
\end{gathered}
$$

If the coded cooperation based relay is not able to decode the message send by secondary source effectively, then it is supposed to be in outage, corresponding to

$$
C_{i, j}=\log _{2}\left(1+\delta_{i, j}\right)<R_{r} / \beta,
$$

where, $\delta_{i, j}$ is the SNR received at node $\{i, j\}$ given by [18]

$$
\delta_{i, j}=\frac{Q g_{i, j}}{\sigma^{2} g_{i, p}},
$$

where $\sigma^{2}$ represent the variance of white Gaussian noise at the secondary receiver.

The SNR received at relay/secondary destination hasprobability density function (PDF) is given by[18]

$$
\begin{gathered}
f_{\gamma_{i, j}}(x)=\int_{0}^{\infty} y f_{Z}(y x) f_{y}(y) d y \\
=\int_{0}^{\infty} y \frac{\lambda_{i, j}}{Q / \sigma^{2}} \exp \left(-\lambda_{i, j} / Q / \sigma^{2}\right) \lambda_{i, p} \exp \left(-\lambda_{i, p} y\right) d y \\
f_{\gamma_{i, j}}(x)=\frac{\lambda_{i, j} \lambda_{i, p} Q / \sigma^{2}}{\left(\lambda_{i, j} x+\lambda_{i, p} Q / \sigma^{2}\right)^{2}} .
\end{gathered}
$$

And the CDF i.e. cumulative distribution function as 


$$
\begin{gathered}
F_{\gamma_{i, j}}(x)=\int_{0}^{x} f_{\gamma_{i, j}}(z) d z=\int_{0}^{x} \frac{\lambda_{i, k} \lambda_{i, p} Q / \sigma^{2}}{\left(\lambda_{i, j} x+\lambda_{i, p} Q / \sigma^{2}\right)^{2}} d z \\
=\frac{\lambda_{i, j} x}{\lambda_{i, j} x+\lambda_{i, p} Q / \sigma^{2}}
\end{gathered}
$$

The proposed system works in two models i.e. primary and enhanced respectively. In primary model only source can transmit its data to the destination through relay and in enhanced model relay can also transmit its data with secondary source to the destination. Both the models are explained in the next sections.

\section{Primary Model}

This section explains basic transmission scheme where source will transmit its data to the destination using coded cooperation based relay node. Frames transmitted in both Phase I and II will be received by destination, for a given secondary sourceas shown in Fig.1. The first frame possesses $\beta$ fraction of the total allocated $N$ bits, while the second frame utilizes the rest of $1-\beta$ fraction of the total bits. In the proposed system, hybridized time sharing method is usedbetween two independent channels.In this way, we can define an outage event as [7]

$$
C_{s, d}\left(\delta_{s, d}\right)=\beta \log _{2}\left(1+\delta_{s, d}\right)+(1-\beta) \log _{2}\left(1+\delta_{s, d}\right)<R_{r}
$$

The average outage probability at the secondary destination is explained by two cases i.e.

\section{Case (a)}

If the code-word sent by secondary source is successfully decoded by relay then conditional outage probability can be written as

$$
\begin{aligned}
P_{\text {out }}^{a}= & \operatorname{Pr}\left(\beta \log _{2}\left(1+\delta_{s, d}\right)+(1-\beta) \log _{2}\left(1+\delta_{r, d}\right)\right)<R_{r} \\
& =\operatorname{Pr}\left(\left(1+\delta_{s, d}\right) \beta_{\left(1+\delta_{r, d}\right)}\right)^{\left.(1-\beta)_{<2} R_{r}\right)}
\end{aligned}
$$

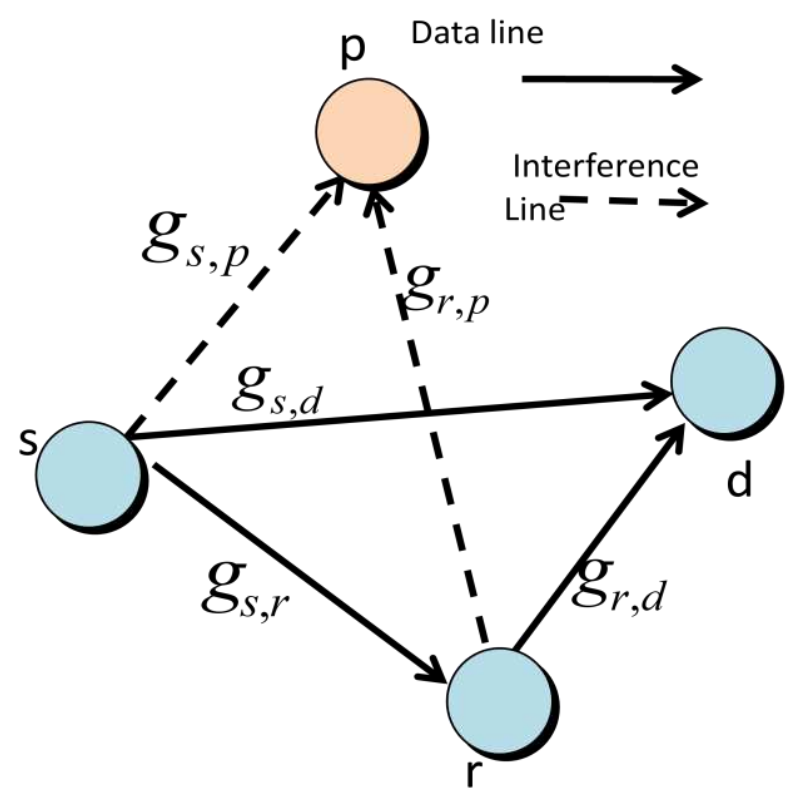

Fig. 1 System model for single relay.

\section{Case (b)}

If relay fails to decode the secondary source's codeword successfully then conditional outage probability is stated as

$$
P_{\text {out }}^{(b)}=\operatorname{Pr}\left(\log _{2}\left(1+\delta_{s, d}<R_{r}\right)\right)=\operatorname{Pr}\left(\delta_{s, d}<2^{R_{r}}-1\right)
$$

For the above two cases average outage probability is

$$
P_{\text {out }}=\operatorname{Pr}\left(\delta_{s, d} \geq 2^{R_{r} / \beta}-1\right) P_{\text {out }}^{(a)}+\operatorname{Pr}\left(\delta_{s, r}<2^{R_{r} / \beta}-1\right) P_{\text {out }}^{(b)}
$$

$$
P_{\text {out }}=\left(\begin{array}{l}
P_{r}\left(\delta_{s, r} \geq 2^{R_{r} / \beta}-1\right) \underbrace{\operatorname{Pr}\left(\left(1+\delta_{s, d}\right)^{\beta}\left(1+\delta_{r, d}\right)^{(1-\beta)}\right)<2}_{H} R_{r} \\
+\operatorname{Pr}\left(\delta_{s, r}<2 / \beta-1\right) \operatorname{Pr}\left(\delta_{s, d}<2^{\left.R_{r}-1\right)}\right)
\end{array}\right)
$$




$$
\begin{aligned}
& P_{\text {out }}=\left(\begin{array}{l}
\left(1-\operatorname{Pr}\left(\delta_{s, r} \leq 2^{R_{r} / \beta}-1\right)\right) H \\
+\operatorname{Pr}\left(\delta_{s, r}<2 R_{r} / \beta-1\right) \operatorname{Pr}\left(\delta_{s, d}<2^{R_{r}}-1\right)
\end{array}\right) \\
& =\left(1-{ }^{2} \int_{0}^{\frac{R_{r}}{\beta}-1} f_{s, r}(x) d x\right) H+\int_{0}^{\frac{R_{r}}{\beta}-1} f_{\gamma_{s, r}}(x) d x \int_{0}^{2} f_{\gamma_{s, d}}(x) d x(17 \\
& P_{\text {out }}=\left[\begin{array}{l}
\left(\begin{array}{c}
\lambda_{s, d}\left(2^{R_{r} / \beta}-1\right) \\
\lambda_{s, r}\left(2^{R_{r} / \beta}-1\right)+\lambda_{s, p} Q / \sigma^{2}
\end{array}\right) H \\
+\frac{\lambda_{s, r}\left(2^{R_{r} / \beta}-1\right)}{\lambda_{s, r}\left(2^{R_{r} / \beta}-1\right)+\lambda_{s, p} Q / \sigma^{2}}\left(\frac{\lambda_{s, d}\left(2^{R_{r}}-1\right)}{\lambda_{s, r}\left(2^{R_{r}}-1\right)+\lambda_{s, p} Q / \sigma^{2}}\right)
\end{array}\right]
\end{aligned}
$$

Where $H$ is given as

$$
\begin{gathered}
H=\operatorname{Pr}\left(\left(1+\delta_{s, d}\right)^{\beta}\left(1+\delta_{r, d}\right)^{(1-\beta)}\right)<2^{R_{r}} \\
H=\underbrace{{ }^{\frac{R_{r}}{\beta}} f_{\gamma_{s, d}}}_{0}(19) \underbrace{\int_{0}^{\int_{0}^{a(x)} \gamma_{r, d}}(y) d y d x}_{\varphi}(20)
\end{gathered}
$$

Where

$$
a(x)=\frac{2^{R_{r} /(1-\beta)}}{(1+x)^{\beta /(1-\beta)}}-1=\frac{2^{R_{r} \rho}}{(1+x)^{\vartheta}}-1
$$

and

$$
\begin{aligned}
& \rho=\frac{1}{(1-\beta)} \\
& \vartheta=\frac{\beta}{(1-\beta)} \\
& \varphi=\frac{A_{r, d} a(x)}{\left(a(x) \frac{A_{r, d}}{Q / \sigma^{2}}+1\right)}
\end{aligned}
$$

where

$$
A_{r, d}=\frac{\lambda_{r, d}}{\lambda_{r, p}}
$$

Substitute the value of $\varphi$ together with the result of equation (9) in equation (20) and then expand itthroughbinomial expansion

$$
(t-1)^{q}=\sum_{r=0}^{q}\left(\begin{array}{l}
q \\
r
\end{array}\right)(-1)^{q-r}(t)^{r}
$$

We can rewrite the equation (20) as

$$
H=\left[\begin{array}{l}
\frac{A_{r, d}}{Q / \sigma^{2}} \sum_{r=0}^{q}\left(\begin{array}{l}
q \\
r
\end{array}\right) 2^{r R_{r} \varphi}(-1)^{-r}\left(2^{R_{r} / \beta}-1\right) \\
F_{1}\left(\begin{array}{l}
\left.1: \vartheta_{r, 2 ; 2 ;-}\left(\frac{2^{\frac{R_{r}}{\beta}}}{-1}\right),-\frac{\left({ }^{\frac{R_{r}}{\beta}}-1\right) A_{s, d}}{Q / \sigma^{2}}\right)
\end{array}\right]
\end{array}\right.
$$

where F1(.) is the Appell hyper geometric function [16]. By using the above obtained value of $H$ in equation (18) the final value of outage probability can be calculated.

\section{III (a). Asymptotic Analysis for Primary Model}

The asymptotic behaviour of the outage probability obtained in the above section is studied by considering high mean SNR value i.e. $\bar{\delta} \rightarrow \infty$.

Here,the average SNR can be stated as

$$
\bar{\delta}_{i, j}=\frac{Q \lambda_{u, v}}{\sigma^{2} \lambda_{u, p}} .
$$

The PDF and CDF expression can be stated as

$$
\begin{gathered}
f_{\bar{\delta}_{i, j}}(x)=\frac{\lambda_{i, j}}{\lambda_{i, p} Q / \sigma^{2}} \\
F_{\bar{\delta}_{i, j}}(x)=\frac{\lambda_{i, j} x}{\lambda_{i, p} Q / \sigma^{2}}
\end{gathered}
$$


The final equation for the asymptotic outage probability $\left(P_{\text {asym }}\right)$ can be illustrated as

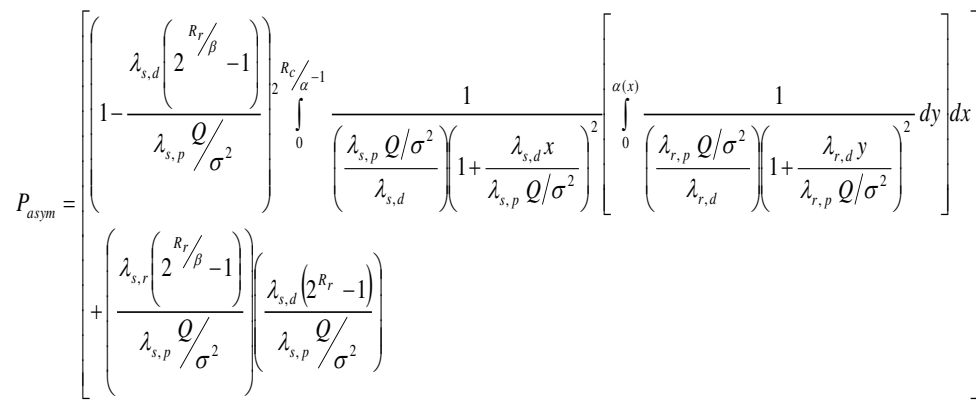

With the help of above equation (27), the asymptotic outage probabilityis calculated. A comparative study of both numerical and asymptotic outage probability is described in the last section.

\section{IV.Enhanced Models}

The cooperative schemes present in literature describes about the scenarios where either source or relay can transmit its information to the destination at any single instant of time as explained in the above section. However, in our enhanced model a pair-wise coded cooperation system is developed where user and relay bothcan act as source simultaneously and transmit their data to the single destination. To make cooperative channel interference free, the data of user and relay is multiplexed by hybridizing FDMA/TDMA. In our proposed system both users i.e. user 1 and 2 can act as a source as well as coded cooperation based relay to each other for transmitting their data. The transmission will take place in two phases. In phase I, the user will transmit their data through orthogonal channels to other user and the destinationsimultaneously. If a user is capable enough to decode the other user's message successfully thenthe second portion of code-word will be transmitted to the destination. If not, then second portion of the code-word will be transmitted by its own in phase II. This cooperative protocol is much easier to apply in practical manner because this requires nofeedback from the relay or knowledge of the $s-r$ channel. Both can transmit their data simultaneously over the same channel in an optimal way which enhances the efficiency of the system and also improves the spectrum utilization. To discuss the proposed system in a rigorous way, all the possible scenarios has been framed for multiuser scheme and their outage probability is calculated in the next section.

\section{Case Wise Outage Probabilities}

In this section, we have developed all the possible transmission scenarios with different sources and their corresponding relays. For the mathematical purpose we define,

$\Delta_{1,2} \cong\left\{\log _{2}\left(1+\delta_{1,2}\right)<R_{r} / \beta\right\}=\left\{\delta_{1,2}<2^{R_{r} / \beta}-1\right\}$

as the event which representsthe occurrence of outage from user 1 to user 2 over the transmission channel, where

$$
\delta_{1,2}=\frac{Q g_{1,2}}{\sigma_{2}^{2} g_{1, p}}=\frac{Q\left|h_{1,2}\right|^{2}}{\sigma_{2}^{2}\left|h_{1, p}\right|^{2}}
$$

and define

$$
\Delta_{2,1} \cong\left\{\log _{2}\left(1+\delta_{2,1}\right)<R_{r} / \beta\right\}=\left\{\delta_{2,1}<2^{R_{r} / \beta}-1\right\}
$$

as the event which representsthe occurrence of outage from user 2 to user 1 over the transmission channel, where

$$
\delta_{2,1}=\frac{Q g_{2,1}}{\sigma_{1}^{2} g_{2, p}}=\frac{Q\left|h_{2,1}\right|^{2}}{\sigma_{1}^{2}\left|h_{2, p}\right|^{2}}
$$

and

$$
P_{b}()=P_{b}\left(\delta_{1,2} \geq 2^{2 R_{r}}-1\right)=1-F_{1,2}\left(2^{2 R_{r}}-1\right)
$$

In this paper, we have developed four scenarios for coded cooperation based multi-user cognitive radio network where both the users can act as source and a relay to each other virtually and can transmit their data simultaneously. The mathematical models of all these cases aredeveloped and their outage probability is also calculated.

\section{Case (a)}

Here, in phase I both of the users can effectively decode the message sent by its partner with the outage probability 


$$
\begin{aligned}
\ell_{a}=P_{b}\left(\Delta_{1}{ }^{c} \bigcap \Delta_{2}{ }^{c}\right) & \\
& =P_{b}\left(\delta_{1,2} \geq 2^{R_{r} / \beta}-1\right) \cdot P_{b}\left(\delta_{2,1} \geq 2^{R_{r} / \beta}-1\right)
\end{aligned}
$$

For this situation, each user in phase Ilis cooperating by sending the remaining portion of the codewordalong with its partner's message as shown in Fig.2. Thus, the user 1 and user 2 have conditional outage probabilities which can be represented as:

$$
\begin{gathered}
p_{\text {out }, 1}^{(a)}=P_{b}\left(\beta \log _{2}\left(1+\delta_{1, d}\right)+(1-\beta) \log _{2}\left(1+\delta_{2, d}\right)<R_{r}\right) \\
=P_{b}\left(\left(1+\delta_{1, d}\right)^{\beta}\left(1+\delta_{2, d}\right)^{(1-\beta)}<2^{R_{r}}\right)
\end{gathered}
$$

and

$$
\begin{gathered}
p_{\text {out }, 2}^{(a)}=P_{b}\left(\beta \log _{2}\left(1+\delta_{2, d}\right)+(1-\beta) \log _{2}\left(1+\delta_{1, d}\right)<R_{r}\right) \\
=P_{b}\left(\left(1+\delta_{2, d}\right)^{\beta}\left(1+\delta_{1, d}\right)^{(1-\beta)}<2^{R_{r}}\right)
\end{gathered}
$$

Primary User

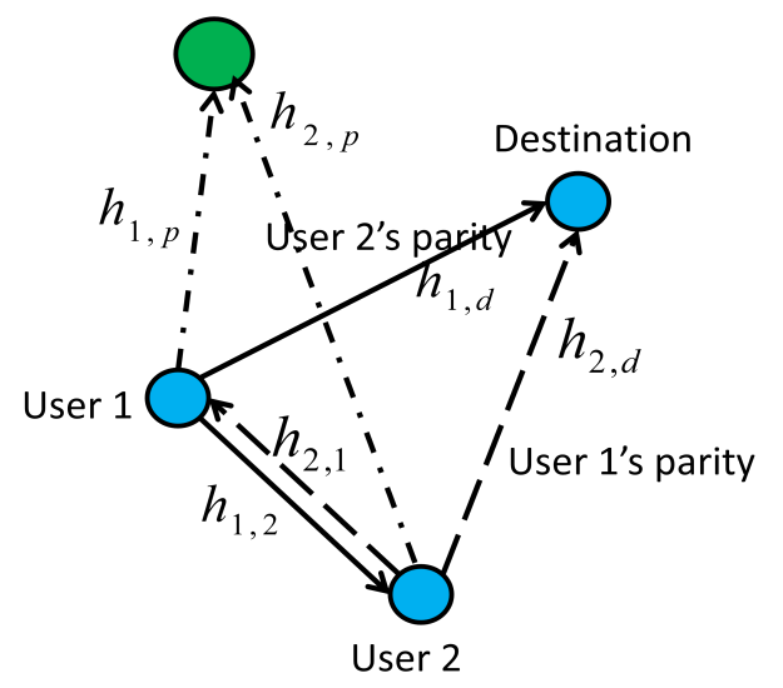

Fig. 2 Both users are cooperating

\section{Case (b)}

In phase I of this case, none of the two users are able to decode message of their partner, so the conditional $P_{\text {out }}$ for this scenario can be represented as

$$
\ell_{b}=P_{b}\left(\Delta_{1,2} \bigcap \Delta_{2,1}\right)=\left\lfloor P_{b}\left(\delta_{1,2}<2^{R_{r} / \beta}-1\right) \cdot P_{b}\left(\delta_{2,1}<2^{R_{r} / \beta}-1\right)\right\rfloor
$$

Fig. 3 explains that no user can effectively decode the messages sent by their partners. So, both users will send the next segment of their own code words by itself in phase II. For user 1 and user 2 the conditional outage probabilities are given as

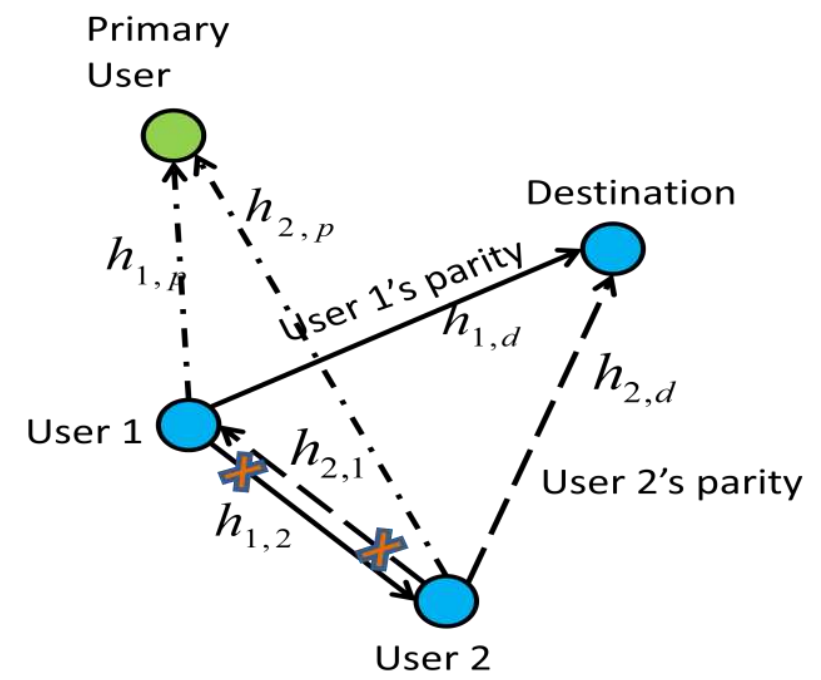

Fig. 3 None of user is cooperating

$$
p_{\text {out }, 1}{ }^{(b)}=P_{b}\left(\beta \log _{2}\left(1+\delta_{1, d}\right)<R_{r}\right)
$$

and

$$
p_{\text {out }, 2}{ }^{(b)}=P_{b}\left(\beta \log _{2}\left(1+\delta_{2, d}\right)<R_{r}\right)
$$

respectively.

\section{Case (c)}

In phase I, user 2 can effectively decode the message send by user 1 , but user 1 itself is unable to successfully decode the message send by user 2 as given in Fig.4. The probability of occurrence of this event is given as

$$
\ell_{c}=P_{b}\left(\Delta_{1,2}{ }^{c} \bigcap \Delta_{2,1}\right)=\left[P_{b}\left(\delta_{1,2} \geq 2^{R_{r} / \beta}-1\right) \cdot P_{b}\left(\delta_{2,1}<2^{R_{r} / \beta}-1\right)\right]
$$

Then, in phase II, second part of user 1's code-word 
is send by both users simultaneously on their respective channels. Decoding of user 2's message at destination is done only by the message received in phase I because none of the user is able to transmit the remaining portion of code-word of user 2 in phase II. Thus, for this case the outage probabilities of both the users can be defined as

$$
\begin{aligned}
p_{\text {out }, 1}{ }^{(c)}= & P_{b}\left(\beta \log _{2}\left(1+\delta_{1, d}\right)+(1-\beta) \log _{2}\left(1+\delta_{1, d}+\delta_{2, d}\right)<R_{r}\right) \\
& =P_{b}\left(\left(1+\delta_{1, d}\right)^{\beta}\left(1+\delta_{1, d}+\delta_{2, d}\right)^{(1-\beta)}<2^{R_{r}}\right) \\
p_{\text {out }, 2}{ }^{(c)} & =P_{b}\left(\beta \log _{2}\left(1+\delta_{2, d}\right)<R_{r}\right)
\end{aligned}
$$

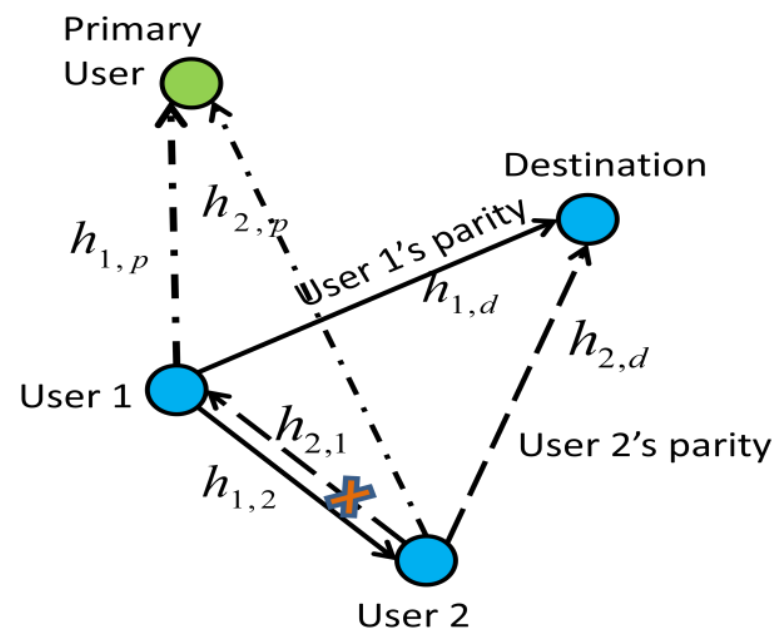

Fig. 4 Only user 2 is cooperating.

\section{Case (d)}

Case (d) and Case (c) both are precisely same here. However the role of user 1 is switched with user 2 and vice-versa as shown in Fig.5. The probability of occurrence of this case is given by

$$
\ell_{d}=P_{b}\left(\Delta_{1,2} \cap \Delta_{2,1}{ }^{c}\right)=\left[P_{b}\left(\delta_{1,2}<2^{R_{r} / \beta}-1\right) P_{b}\left(\delta_{2,1} \geq 2^{R_{r} / \beta}-1\right)\right]
$$

The conditional $P_{\text {out }}$ for user 1 and user 2 are defined as:

$$
\begin{gathered}
p_{\text {out }, 1}{ }^{(d)}=P_{b}\left(\beta \log _{2}\left(1+\delta_{1, d}\right)<R_{r}\right) \\
P_{\text {out }, 2}{ }^{(d)}=P_{b}\left(\beta \log _{2}\left(1+\delta_{1, d}\right)+(1-\beta) \log _{2}\left(1+\delta_{1, d}+\delta_{2, d}\right)<R_{r}\right) \\
=P_{b}\left(\left(1+\delta_{2, d}\right)^{\beta}\left(1+\delta_{1, d}+\delta_{2, d}\right)^{(1-\beta)}<2^{R_{r}}\right)
\end{gathered}
$$

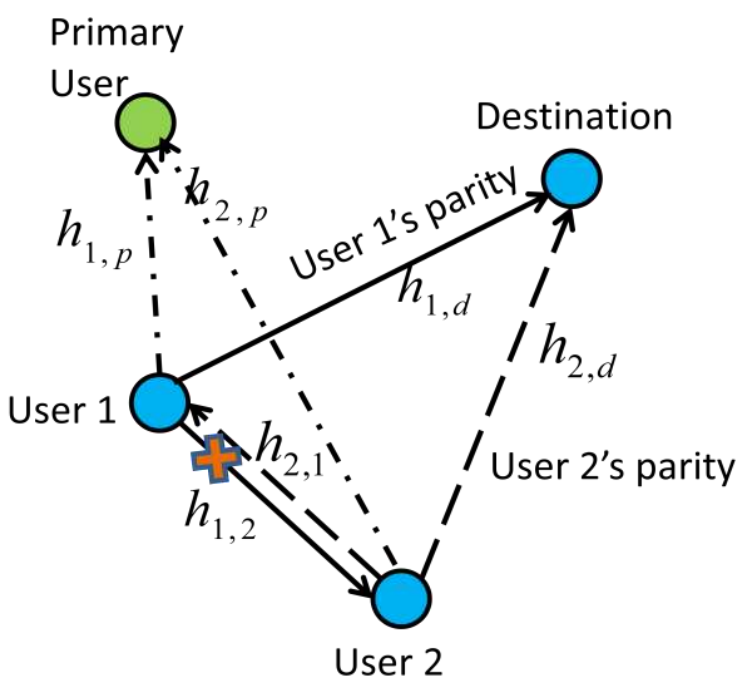

Fig. 5 Only user 1 is cooperating.

Using all above scenarios the final average $P_{\text {out }}$ of user $\mathrm{i}$, for $\mathrm{i} €\{1,2\}$, can be calculated as

$$
\begin{aligned}
& \text { Pout }=\ell_{a} * P_{\text {out }, i}{ }^{(a)}+\ell_{b} * P_{\text {out }, i}{ }^{(b)}+\ell_{c} * P_{\text {out }, i}{ }^{(c)}+\ell_{d} * P_{\text {out }, i}{ }^{(d)} \\
& =\left[P_{b}\left(\delta_{1,2} \geq 2^{R_{r} / \beta}-1\right) P_{b}\left(\delta_{2,1} \geq 2^{R_{r} / \beta}-1\right)\right] \\
& *\left[P_{b}\left(\left(1+\delta_{1, d}\right)^{\beta}\left(1+\delta_{2, d}\right)^{(1-\beta)}<2^{R_{r}}\right)+P_{b}\left(\left(1+\delta_{2, d}\right)^{\beta}\left(1+\delta_{1, d}\right)^{(1-\beta)}<2^{R_{r}}\right)\right] \\
& +\left[P_{b}\left(\delta_{1,2}<2^{R_{r} / \beta}-1\right) P_{b}\left(\delta_{2,1}<2^{R_{r} / \beta}-1\right)\right] \\
& *\left[P_{b}\left(\delta_{1, d}<2^{R_{r} / \beta}-1\right)+P_{b}\left(\delta_{2, d}<2^{R_{r} / \beta}-1\right)\right] \\
& +\left[P_{b}\left(\delta_{1,2} \geq 2^{R_{r} / \beta}-1\right) \cdot P_{b}\left(\delta_{2,1}<2^{R_{r} / \beta}-1\right)\right] \\
& *\left[P_{b}\left(1+\delta_{1, d}\right)^{\beta}\left(1+\delta_{1, d}+\delta_{2, d}(1-\beta)<2^{R_{r}}\right)+P_{b}\left(\left(1+\delta_{2, d}\right)^{\beta}<2^{R_{r}}\right)\right] \\
& +\left[P_{b}\left(\delta_{1,2}<2^{R_{r} / \beta}-1\right) P_{b}\left(\delta_{2,1} \geq 2^{R_{r} / \beta}-1\right)\right] \\
& *\left[P_{b}\left(\left(1+\delta_{1, d}\right)^{\beta}<2^{R_{r}}\right)+P_{b}\left(\left(1+\delta_{2, d}\right)^{\beta}\left(1+\delta_{1, d}+\delta_{2, d}\right)^{(1-\beta)}<2^{R_{r}}\right)\right]
\end{aligned}
$$

The final expression for $P_{\text {out }}$ will be obtained by substituting the values of equation (29) and (31) in equation (45) which will finally represent the outage probability of the multi-user system. 


\section{V(a). Asymptotic Analysis for Enhanced Model}

To validate the results we have used the asymptotic analysis. Here, we assume that SNR is very high in value i.e.

$$
\gamma \rightarrow \infty
$$

Then the value of average SNR is:

$$
\bar{\delta}_{u, v}=\frac{Q \lambda_{u, v}}{\sigma^{2} \lambda_{u, p}}
$$

The corresponding expressions for asymptotic PDF and $\mathrm{CDF}$ are derived as

$$
\begin{gathered}
f_{\bar{\gamma}}=\frac{\lambda_{u, v}}{\lambda_{u, p} Q / \sigma^{2}}, \\
F_{\bar{\gamma}_{u, v}}(x)=\frac{\lambda_{u, v} x}{\lambda_{u, p} Q / \sigma^{2}}
\end{gathered}
$$

The final equation for the $P_{\text {asym }}$ is obtained by using above mentioned PDF and CDF equation (47) in outage probability equation (45). To improve the validation of the proposed system, asymptotic analysis has been done and the collective results are compared in next section.

\section{VI.Numerical Results}

This portion of research paper explains the numerical results of both the primary and enhanced coded cooperation based CR system model. The deviation in $P_{\text {out }}$ in form of channel gain and interference temperature constraint, $Q$ is illustrated in this section. Asymptotic behaviour of both the models is also plotted to validate the numerical results. For the ease of illustration, we assume all the channels to be identically distributed with $\lambda_{\mathrm{s}, \mathrm{r}}=\lambda_{\mathrm{r}, \mathrm{d}}=\lambda_{\mathrm{s}, \mathrm{d}}=1$ and $\mathrm{R}=1 \mathrm{bits} / \mathrm{sec}, \sigma^{2}=0 \mathrm{~dB}$ and $\mathrm{K}=\beta=$ $1 / 2$.

The variation of outage probability with interference temperature constraint, $Q$ is shown in Fig.6. The $P_{\text {out }}$ graph is drawn for wide range of $Q$ i.e $5 \mathrm{~dB}$ to $20 \mathrm{~dB}$ for different values of channel gain parameter. The primary system model having single relay shows a noticeable improvement in outage probability from $10^{0.8}$ at $Q=5 \mathrm{~dB}$ to $10^{-3.5}$ at $Q=20 \mathrm{~dB}$. More interestingly a remarked improvement is noted in outage probability of system even for single relay coded cooperation at higher value of interference temperature constraint, $Q$ because secondary user will be allowed to transmit high power for higher value of $Q$. To validate the analysis asymptotic results are also plotted on the same graph which are coinciding at higher values of interference temperature constraint, $Q$ and this proves that the analysis is quite accurate.

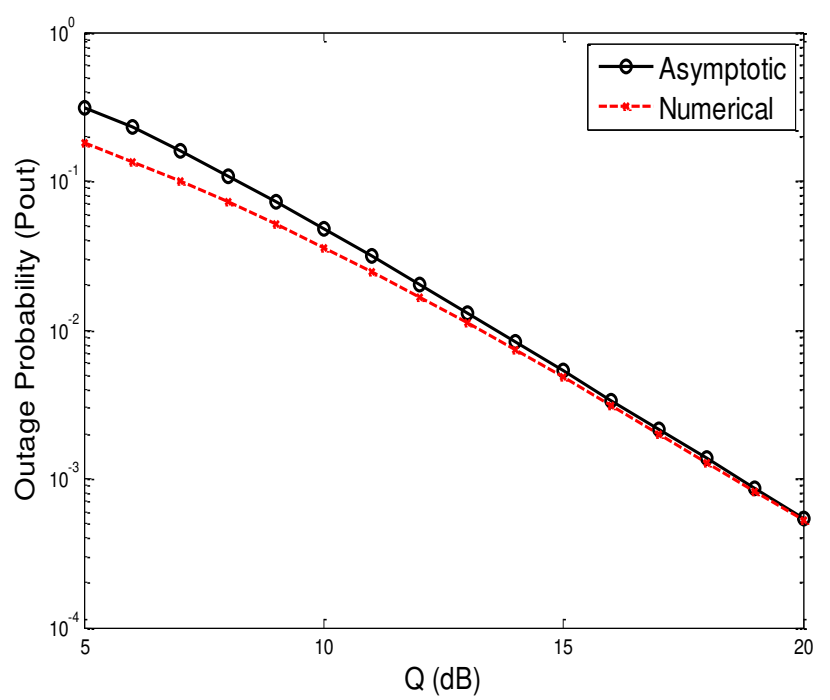

Fig. 6 Outage probability w.r.t ' $Q$ '

The Fig. 7 represents the variation of $P_{\text {out }}$ versus relay and primary user channel gain parameter, $\lambda_{\mathrm{r}, \mathrm{p}}$ for interference temperature, i.e. $Q=20 \mathrm{~dB}$. The graph shows that for low value of $\lambda_{r, p}$, the $P_{\text {out }}$ is not notablychanged, but at higher values of $\lambda_{\mathrm{r}, \mathrm{p}}$ there is a considerableenhancement and outage probability reduces to $10^{-3.5}$. To validate the analysis asymptotic results are also plotted on the same graph which are coinciding at higher values of channel gain parameter, $\lambda_{\mathrm{r}, \mathrm{p}}$ that further proves the correctness of the analysis. 


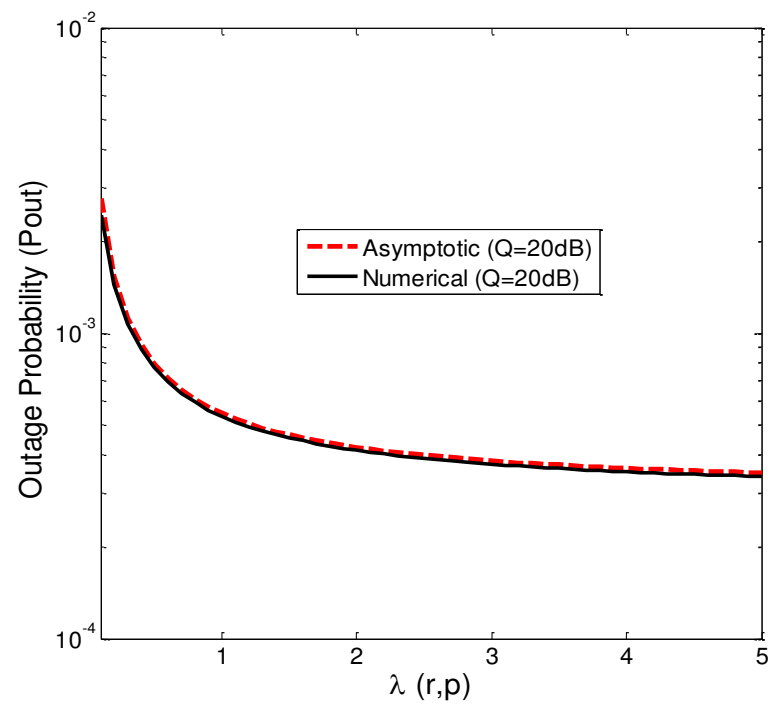

Fig. 7 Outage probability w.r.t to $\lambda_{\mathrm{r}, \mathrm{p}}$

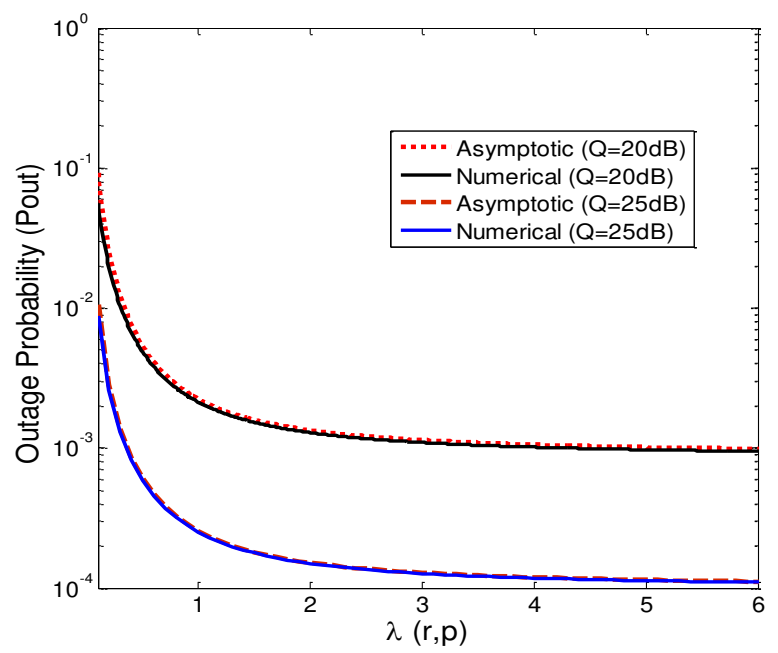

Fig.8 Outage probability w.r.t. channel gain parameter $\lambda_{r, p}$ at different interference temperature constraint, $Q$

The results in Fig.8 shows the effect of relay and primary user channel gain parameter, $\lambda_{r, p}$ on outage probability for the enhanced model for wide range of interference temperature, i.e. $Q=20 \mathrm{~dB}$ and $25 \mathrm{~dB}$. Results in Fig.8 shows that the outage probability improves from $10^{-1}$ to $10^{-2}$ with the increase of $\lambda_{r, p}$ and are constant for the higher value of $\lambda_{r, p}$. So, the result proves that the designed system can enhance the diversity and communication quality even for
Rayleigh channel. The plot illustrates that at higher values of $Q \mathrm{~dB}$, the numerical as well as asymptotic results of outage probability coincides.

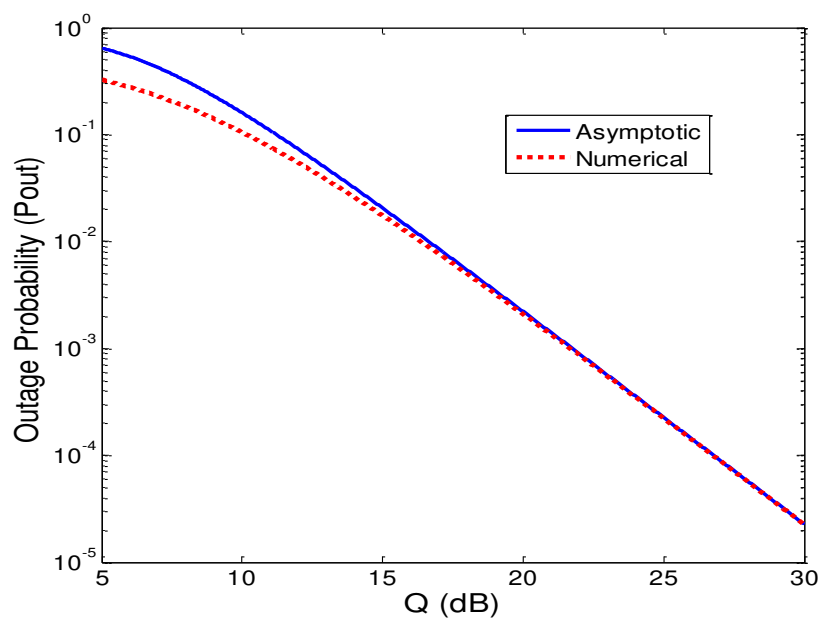

Fig.9 Outage probability versus interference temperature constraint, $Q$

In Fig.9, the results for outage probability are taken for enhanced model with wide range of interference temperature , $Q \mathrm{~dB}$ values i.e. $5 \mathrm{~dB}$ to $30 \mathrm{~dB}$ for both users. Results demonstrate that the system performance improves with an increase of the interference temperature constraint because higher values of $Q \mathrm{~dB}$ makes secondary user to transmit more power. As a result, the outage probability improves from $10^{0}$ at $Q=5 \mathrm{~dB}$ to $10^{-5}$ at $Q=$ 30dB.This is a marked improvement in bandwidth efficiency and spectrum utilization. Moreover, both the usersshow the same outage behaviour and result is also validated with asymptotic analysis.

\section{Conclusion}

Coded cooperation is an effective protocol for achieving transmission diversity for the singleantenna system. We have developed an optimal multi-user system using underlay based cognitive radio which will provide diversity, spectrum and bandwidth gains. Finally, a hierarchical mathematical model for the purposed system is developed and performance is evaluated in terms of outage probability. The functioning of the system is also analyzed in the form of interference temperature constraint and channel gain via 
simulation and asymptotic analysis. The proposed system model can work as benchmark technology for all those cognitive radio applications which require better spectrum efficiency even if having the constraint of size and multiple physical antennas.

\section{Declarations}

Not Applicable

\section{References}

[1] Goldsmith, A. J., Jafar, S. A., Maric, I., \&Srinivasa, S. (2009). Breaking spectrum gridlock with cognitive radios: An information theoretic perspective. Proceedings of the IEEE, 97(5), 894914.

[2] Devroye, N., Mitran, P., \&Tarokh, V. (2006). Achievable rates in cognitive radio channels. IEEE Transactions on information theory, 52(5), 18131827.

[3] Jafar, S. A., \&Srinivasa, S. (2007). Capacity limits of cognitive radio with distributed and dynamic spectral activity. IEEE Journal on selected Areas in Communications, 25(3), 529-537.

[4] Akin, S., \&Gursoy, M. C. (2010). Effective capacity analysis of cognitive radio channels for quality of service provisioning. IEEE Transactions on Wireless Communications, 9(11), 3354-3364.

[5] Haykin, S. (2005). Cognitive radio: brainempowered wireless communications. IEEE journal on selected areas in communications, 23(2), 201220.

[6] W. Krenik, A. M. Wyglinsky and L. Doyle, "Cognitive radios for dynamic spectrum access," IEEE Commun. Mag., vol. 45, no. 5, pp. 64-65, May 2007.

[7] Aoki, H., Takeda, S., Yagyu, K., \& Yamada, A. (2006). IEEE $802.11 \mathrm{~s}$ wireless LAN mesh network technology. NTT DoCoMo Technical Journal, 8(2), 13-21.

[8] Das, P., Mehta, N. B., \& Singh, G. (2015). Novel relay selection rules for average interferenceconstrained cognitive AF relay networks. IEEE Transactions on Wireless Communications, 14(8), 4304-4315.
[9] M. Xia and S. Aïssa, "Cooperative AF relaying in spectrum-sharing systems: Performance analysis under average interference power constraints and Nakagami-m fading," IEEE Trans. Commun., vol. 60, no. 6, pp. 1523-1533, Jun. 2013.

[10] Xia, M., \&Aissa, S. (2012). Cooperative AF relaying in spectrum-sharing systems: Outage probability analysis under co-channel interferences and relay selection. IEEE Transactions on Communications, 60(11), 3252-3262.

[11] Xia, M., \&Aissa, S. (2014). Spectrum-sharing multi-hop cooperative relaying: Performance analysis using extreme value theory. IEEE Transactions on Wireless Communications, 13(1), 234-245.

[12] Chen, J., Si, J., Li, Z., \& Huang, H. (2012). On the performance of spectrum sharing cognitive relay networks with imperfect CSI. IEEE Communications Letters, 16(7), 1002-1005.

[13] Zhong, C., Ratnarajah, T., \& Wong, K. K. (2011). Outage analysis of decode-and-forward cognitive dual-hop systems with the interference constraint in Nakagami-m fading channels. IEEE Transactions on Vehicular Technology, 60(6), 28752879.

[14] Moualeu, J. M., Hamouda, W., \&Takawira, F. (2017). Cognitive coded cooperation in underlay spectrum-sharing networks under interference power constraints. IEEE Transactions on Vehicular Technology, 66(3), 2099-2113.

[15]Singh, G., Kaur, G., Dwivedi, V. K., \&Yadav, P. K. (2018). Development of coded-cooperation based multi-relay system for cognitive radio using mathematical modeling and its performance analysis. Wireless Networks, 1-7.

[16] Gradshteyn, I. S., \&Ryzhik, I. M. (2007). Table of integrals,series, and products (3rd ed.). New York, NY: Academic.

[17] Moualeu, J. M., Hamouda, W., \&Takawira, F. (2017). Cognitive coded cooperation in underlay spectrum-sharing networks under interference power constraints. IEEE Transactions on Vehicular Technology, 66(3), 2099-2113.

[18] Guo, Y., Kang, G., Zhang, N., Zhou, W., \& Zhang, P. (2010). Outage performance of relayassisted cognitive-radio systemunder spectrum- 
sharing constraints. Electronics Letters, 46(2),182184. 